\title{
Role of human tissue kallikrein in gastrointestinal stromal tumour invasion
}

\section{P Dominek 1,6, P Campagnolo 1,6, M H-Zadeh², N Kränkel', M Chilosi', JA Sharman ${ }^{4}$, A Caporali', G Mangialardi', G Spinetti ${ }^{5}$, C Emanueli', M Pignatelli ${ }^{2}$ and $\mathbf{P}$ Madeddu*,I}

'Experimental Cardiovascular Medicine, Bristol Heart Institute, University of Bristol, Level 7, Bristol Royal Infirmary, Upper Maudlin Street, Bristol, UK; ${ }^{2}$ Clinical Science at South Bristol, University of Bristol, Level 7, Bristol Royal Infirmary, Upper Maudlin Street, Bristol, UK; ${ }^{3}$ University of Verona, Verona, Italy;

${ }^{4}$ Vantia Ltd, Southampton Science Park, Southampton, UK; ${ }^{5}$ Multimedica IRCCS, Milan, Italy

BACKGROUND: Human tissue kallikrein (hKI) generates vasodilator kinins from kininogen and promotes angiogenesis by kinin-dependent and kinin-independent mechanisms. Here, we investigate the expression and functional relevance of hKI in human gastrointestinal stromal tumour (GIST).

METHODS: Vascularisation and hKI expression of GIST samples were assessed by immunohistochemistry. In two GIST cell lines, hKI expression was assessed by PCR, and hKI protein levels and activity were measured by ELISA and an amidolytic assay, respectively. The effect of hKI silencing, inhibition or overexpression on GIST cell proliferation, migration and paracrine induction of angiogenesis was studied. Finally, local and systemic levels of hKI were assessed in mice injected with GIST cells.

RESULTS: Human tissue kallikrein was detected in 19 out of 22 human GIST samples. Moreover, GIST cells express and secrete active hKI. Titration of hKI demonstrated its involvement in GIST invasive behaviour, but not proliferation. Furthermore, hKI released by GIST cells promoted endothelial cell migration and network formation through kinin-dependent mechanisms. Gastrointestinal stromal tumour implantation in nude mice resulted in local and systemic hKI expression proportional to tumour dimension.

CONCLUSIONS: Human tissue kallikrein is produced and released by GIST and participates in tumour invasion. Further studies are needed to validate hKI as a diagnostic biomarker and therapeutic target in GIST.

British Journal of Cancer (2010) I 03, |422- |431. doi:I0.1038/sj.bjc.6605906 www.bjcancer.com

Published online 21 September 2010

(c) 2010 Cancer Research UK

Keywords: kallikrein; angiogenesis; invasion; GIST

Gastrointestinal stromal tumour (GIST) is one of the most common mesenchymal tumours of the gastrointestinal tract, the clinical features of which comprise acute or chronic bleeding, altered bowel function and intestinal obstruction or perforation, depending on the size and location of the tumour (Miettinen and Lasota, 2006). Approximately $85-90 \%$ of GISTs harbour activating mutations for the stem cell factor (SCF) receptor CD117 (c-Kit) or the alpha-type platelet-derived growth factor receptor (PDGFR $\alpha$ ) (Heinrich et al, 2000; Hirota et al, 2000), which makes this tumour responsive to the tyrosine kinase inhibitor imatinib mesylate (Demetri et al, 2002). However, the mechanisms involved in the invasive capacity of GIST remain largely unknown.

The kallikrein (hK) family has been recognised to have fundamental roles in cancer and vascular biology (Bhoola et al, 2001; Borgono and Diamandis, 2004; Clements et al, 2004; Madeddu et al, 2007). Individual members of the hK family have in the past been identified as biomarkers for cancer, such as prostate cancer-specific marker hK3 (Welch and Albertsen, 2009).

*Correspondence: Dr P Madeddu; E-mail: madeddu@yahoo.com

${ }^{6}$ These authors contributed equally to the study.

Received II May 2010; revised 18 August 2010; accepted 19 August 20I0; published online 2I September 2010
To date, no studies have investigated the involvement of hKs in the growth and development of GIST.

Human tissue kallikrein (hK1) has a crucial role in postischaemic neovascularisation (Emanueli et al, 2000, 2001, 2004; Yao et al, 2008; Stone et al, 2009). Furthermore, hK1 has been implicated in the growth and invasiveness of pancreatic carcinoma (Wolf et al, 2001), oesophageal carcinoma (Dlamini et al, 1999; Dlamini and Bhoola, 2005), gastric malignancy (Sawant et al, 2001) and lung cancer (Chee et al, 2008). Although the exact molecular mechanisms by which hK1 promotes tumour growth and invasion have not been determined so far, two principal actions of hK1 may have a role: (1) promoting tumour cell invasion of extracellular matrix by its protease activity, directly or through the activation of metalloproteinases (MMPs) (Tschesche et al, 1989; Desrivieres et al, 1993; Menashi et al, 1994; Leeb-Lundberg et al, 2005; Stone et al, 2009) and (2) activating kinin receptors, either directly (Biyashev et al, 2006) or through the generation of kinins (Madeddu et al, 2007). Autocrine activation of the kinin B2 receptor (B2R) on tumour cells may promote proliferation and motility, whereas paracrine action could induce endothelial cell (EC) proliferation and migration, thus increasing tumour vascularisation.

In this study, we aimed to investigate whether hK1 is expressed and released by GIST and participates in tumour growth and expansion. 


\section{MATERIALS AND METHODS}

\section{Immunohistochemistry}

Formalin-fixed and paraffin-embedded GIST specimens were obtained from the Departments of Pathology of Bristol University (UK) and Verona University (Italy), with the approval of the local ethics committee. Xenograft tumour samples were obtained after excision from euthanised animals. Serial sections $(3 \mu \mathrm{m})$ were incubated overnight with monoclonal antibodies antihuman hK1 $(1: 100, \mathrm{R} \& \mathrm{D})$ and CD31 ( $1: 40, \mathrm{DAKO})$ or Isolectin B4 $(1: 100$, Sigma, St Louis, MO, USA) at $4{ }^{\circ} \mathrm{C}$. Immunoreactivity was detected using the $\mathrm{ABC}$ method (Vector Labs, Burlingame, CA, USA) or by applying a suitable fluorescence-labelled secondary antibody. Normal mouse IgG (Santa Cruz, Santa Cruz, CA, USA) was used as a negative control.

Images were taken at $\times 200$ and $\times 400$ magnification using an Olympus light microscope (BX 40, Southall, UK). The stained area and intensity score were evaluated in the GNU Image Manipulation Program (GIMP) using colour selection and histogram function. HK1 staining intensity was recalculated to a $0-100 \%$ scale, where zero represents no staining and 100 represents the darkest (black) area. In CD31-stained samples, vessels were expressed as the average number of vessels per section.

\section{GIST cell line}

The immortalised GIST882 and GIST48 cell lines were a kind gift from Dr J Fletcher (Brigham and Women's Hospital, Boston, MA, USA) (Tuveson et al, 2001). GIST882 was cultured in RPMI 1640

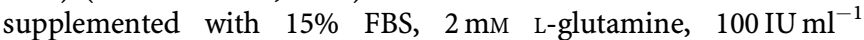
penicillin and $100 \mu \mathrm{g} \mathrm{ml}^{-1}$ streptomycin (Cambrex, East Rutherford, NJ, USA). GIST48 was cultured in F10 (GIBCO) with 20\% FBS, 2 mM L-glutamine, $100 \mathrm{IU} \mathrm{ml}^{-1}$ penicillin and $100 \mu \mathrm{g} \mathrm{ml}^{-1}$ streptomycin, MITO serum extender and bovine pituitary extract (BD).

Human umbilical vein endothelial cells (HUVEC, Cambrex) were grown in EGM-2 containing 2\% FBS (Lonza, Basel, Switzerland).

\section{Conditioned medium}

Highly confluent GIST882 or GIST48 cells were incubated with serum-free medium for $24 \mathrm{~h}$. After medium collection, cells were trypsinised and counted to establish total number.

\section{hK1 silencing}

Three to five million GIST882 cells were transfected with $150 \mathrm{~nm}$ of small interfering RNA (siRNA) for hK1 or scrambled control using Amaxa electroporation protocol (Amaxa Biosystems, Gaithersburg, MD, USA). Effective silencing was verified by RTPCR and ELISA (see below). On-target plus siRNA was purchased from Dharmacon (Lafayette, CO, USA).

\section{Infection with adenoviral vector carrying hK1}

GIST882 cells were incubated with 50 moi (multiplicity of infection) of Ad.hK1 or Ad.Null overnight. Experiments were performed $24 \mathrm{~h}$ later. Successful infection was verified by RT - PCR (see below). Adenoviruses were prepared as previously described (Stone et al, 2009).

\section{Measurement of hK1 levels and activity}

Immunoreactive hK1 in cell culture supernatants and blood was measured by ELISA as previously reported (Wang et al, 1995; Porcu et al, 2002, 2004) and hK1 activity was assayed using the chromogenic substrate S-2266, following the manufacturer's instructions (Chromogenix, Milano, Italy) and as previously described (Madeddu et al, 1993).

\section{Expression analysis}

RNA was obtained using an RNeasy Minikit (Qiagen, Crawley, UK) and reverse transcribed with MMLV (Invitrogen, Paisley, UK). Real-time PCR was carried out with PCR Mastermix 2x (Promega, Madison, WC, USA).

Primers were designed for hK1: Forward $5^{\prime}$-GGGTCGCCACAAC TTGTTTG- $3^{\prime}$ and Reverse 5'-GCTGTAGTCCTCGTCTGCTT-3'; B1R: Forward $5^{\prime}$-CTTCCCTCAAAATGCTACGGC- $3^{\prime}$ and Reverse $5^{\prime}$-TCTGCCACGTTCAGTTGCC-3'; B2R: Forward 5'-GTCTGTTCG TGAGGACTCCG- $3^{\prime}$ and Reverse $5^{\prime}$-CTGGGCAAAGGTCCCGTTA AG-3'; ACE: Forward 5'-AACGAAACCCACTTTGATGC-3' and Reverse 5'-TCAGCCTCATCAGTCACCAG-3'; HMK: Forward $5^{\prime}$-TGGGGCCATGAAAAACAAAG- $3^{\prime}$ and Reverse $5^{\prime}$-CTTGGCTAG GGAAGGGATGG-3'; LMK: Forward $5^{\prime}$-CCAGCATCTGAGAGGG AGGT- $3^{\prime}$ and Reverse $5^{\prime}$-GCAGAATGGGTAGGGCTGAA- ${ }^{\prime}$.

\section{BrdU cell proliferation assay}

Gastrointestinal stromal tumour cells were seeded in 96-well plates (5000 cells per well) and incubated with BrdU for $24 \mathrm{~h}$ in normal medium or in the presence of $0.5,0.05$ or $0.005 \mu \mathrm{M}$ of specific hK1 inhibitors VA999154 or VA999024, kindly provided by Vantia Ltd. (Chilworth, UK) BrdU incorporation was determined following the manufacturer's protocol (Roche, Melwin, UK).

\section{GIST invasion assay}

The invasion capacity of GIST882 and GIST48 cells was measured by seeding $5 \times 10^{4}$ cells on top of an $8 \mu \mathrm{m}$ filter coated with Matrigel (BD, 1:100). Medium containing 5\% FBS was used as a stimulus. hK1 inhibitors VA999154 and VA999024 were added at a concentration of $0.5 \mu \mathrm{M}$ as described above. After $24 \mathrm{~h}$, the filters were mounted with DAPI to recognise the nuclei of migrated cells. Cell number was calculated by averaging the counts of five microscopic fields (photographed at $\times 20$ ).

\section{Endothelial cell migration assay}

The migratory response of ECs to GIST was assessed in a transwell array (Corning, Corning, NY, USA). Briefly, $3 \times 10^{5}$ GIST882 cells were plated on the bottom chamber of the migration system. Human umbilical vein ECs (HUVECs) were preincubated with $B_{1} R$ or $B_{2} R$ antagonists (Lys-des-Arg ${ }^{9} \mathrm{Leu}^{8}-\mathrm{BK}$, LdL-BK or Icatibant, IC, respectively, $2 \times 10^{-7} \mathrm{M}$ ) or PBS (vehicle), plated on top of the insert (50000 cell per insert) and then left to migrate overnight in the presence or absence of $B_{1} R$ or $B_{2} R$ antagonists. Migrated HUVECs were counted as described for the invasion assay and the percentage of migrated cells was calculated on the number of plated cells.

\section{Matrigel angiogenesis assay}

HUVECs pretreated for 30 min with the hK1 inhibitor kallistatin (1 $\mu \mathrm{M}, \mathrm{R} \& \mathrm{D})$ (Wolf et al, 1999), the serine protease inhibitor Aprotinin $\left(50 \mathrm{U} \mathrm{ml}^{-1}\right.$, Bayer, FRG) or vehicle were plated on growth factor-reduced Matrigel (BD Biosciences, Erembodegem, Belgium) in the presence of GIST882-conditioned medium or unconditioned medium (as a control). Total tube length and average tube thickness were measured on photographs captured at $24 \mathrm{~h}$ and analysed using ImagePro Plus software (Media Cybernetics, Bethesda, MD, USA).

\section{FACS analysis}

Cells were incubated with rabbit polyclonal antibodies for $B_{1} R$ and $B_{2} R$ (1:100, Sigma), followed by FITC-labelled antirabbit secondary antibodies (Sigma) and analysed in a FACScalibur (BD Biosciences) flow cytometer. Controls were stained with isotype control. 


\section{Xenograft model}

The experiments involving mice were conducted in accordance with the Guide for the Care and Use of Laboratory Animals prepared by the Institute of Laboratory Animal Resources and with previous approval of the UK Home Office and the University of Bristol ethics committee.

GIST882 cells were implanted under the dorsal skin of BALB/c nude mice in three doses $\left(3 \times 10^{6}-3 \mathrm{M}, 5 \times 10^{6}-5 \mathrm{M}\right.$ and $8 \times 10^{6}-8 \mathrm{M}$ cells or vehicle; four mice per dosage). Tumour growth was monitored biweekly. All mice were euthanised with an excess of anaesthesia when the tumour size of the highest-dose group reached $16 \mathrm{~mm}^{3}$. Before being killed, blood was collected by cardiac puncture and tumour tissue was collected for further analysis.

\section{Statistical analysis}

Continuous variables were compared by one-way ANOVA or Student's $t$-test as appropriate. Relationships between variables were determined by the Pearson correlation coefficient. Continuous data are expressed as mean \pm s.e.m. A $P$ value $<0.05$ was considered statistically significant. Analyses were performed with GraphPad Prism 5.0 (Graphpad software, La Jolla, CA, USA).

\section{RESULTS}

\section{High circulating levels of $\mathrm{hK} 1$ in a GIST patient}

We previously reported that hK1 levels are remarkably elevated in peripheral blood of patients with critical carotid artery obstruction and normalised after endarterectomy (Porcu et al, 2004). Only one subject of this series showed persistently high hK1 levels before and after revascularisation (1456 and $1681 \mathrm{pg} \mathrm{ml}^{-1}$, respectively). The patient was referred to us 8 months after endarterectomy because of the appearance of constipation. Computerised tomography scanning documented a solid mass $(7 \times 6 \mathrm{~cm}$ diameters $)$ infiltrating the ileum and reaching the abdominal wall (Figure 1A). At power Doppler, the mass appeared irregularly perfused (Figure 1B). Surgical resection resulted in a remarkable reduction of circulating hK1 (368 $\mathrm{pg} \mathrm{m}^{-1}, 2$ months after surgery). Histological examination of the mass revealed the characteristics of mixed spindle and epithelioid cell GIST, positive for c-Kit and negative for S-100 protein, glial fibrillary acidic protein (GFAP), desmin and CD34 (not shown). Vascular cells (EC) and GIST cells were positive for hK1 (red arrows, Figure 1C). The vascular endothelium was identified in consecutive sections by positive staining for von Willebrand factor (Figure 1D). Specificity of the reaction was confirmed by parallel staining of hK1-producing salivary glands and GIST specimens (Figure $1 \mathrm{E}$ and F).
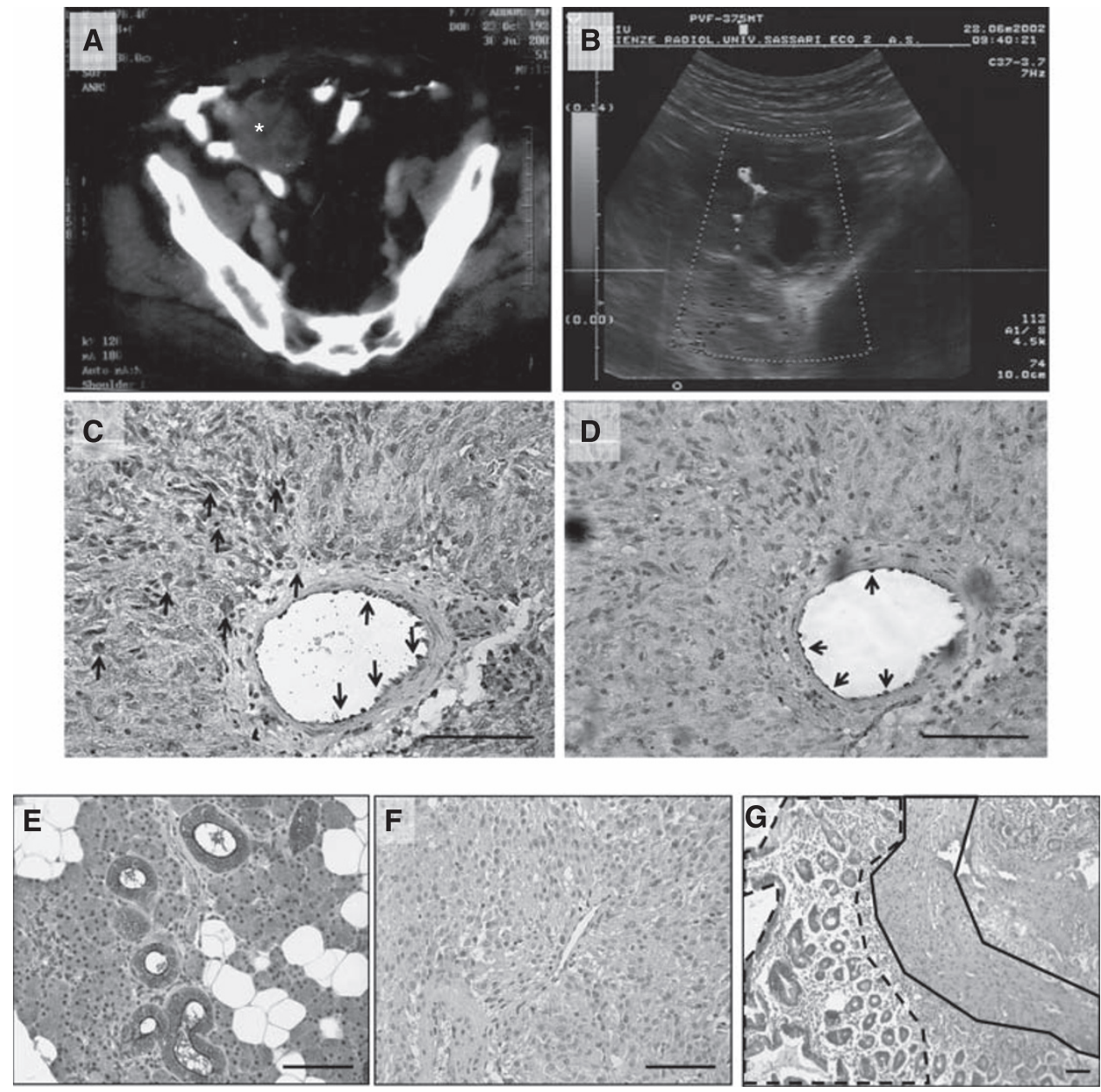

Figure I Clinical case. Representative CT scan images showing the presence of a tumour mass, highlighted by an asterisk, in the abdominal cavity (A). The mass appears irregularly perfused, as shown by Doppler imaging (B). Immunohistochemistry analysis confirmed typical GIST characteristics and expression of hKI (red arrows) in tumour cells (C) and vasculature (D, stained with von Willebrand). Specificity of the hKI staining was confirmed on sections of salivary glands (E, positive control) and GIST incubated with mouse lgG (F, negative control). Biopsies derived from normal gastrointestinal tract reveal no expression of hKI in either the epithelial layer (dashed line) or in the muscular area (continuous line) (G). Scale bar: $100 \mu \mathrm{m}$. 
Normal gastrointestinal tissue was negative, thus confirming the aberrant expression of hK1 by GIST (Figure 1G).

\section{Retrospective analysis of $\mathrm{hK} 1$ expression in GIST}

We verified the expression of hK1 in a series of 22 GIST cases. Patient characteristics are summarised in Table 1, which also

Table I Patients and tumours characteristics

\begin{tabular}{lc}
\hline Number of cases & 22 \\
Average age (years) & $60 \pm 2$ \\
Sex & \\
Male & 11 \\
Female & 11 \\
& \\
Location & 13 \\
Stomach & 7 \\
Small bowel & 2 \\
Rectum & \\
& \\
Risk level & 5 \\
Low & 9 \\
Intermediate & 8 \\
High & \\
Main cell type & \\
Spindle & 13 \\
Epithelioid & 8 \\
Mixed & 1 \\
\hline
\end{tabular}

reports tumour localisation and risk of aggressive behaviour as proposed by Fletcher et al (Fletcher et al, 2002). Immunohistochemistry revealed that 19 of 22 GIST cases were positive for hK1 with variable intensity and percentage of positive tissue (representative microphotographs in Figure 2A and B).

Quantitative assessment of hK1 expression was performed by measuring (1) the section area positive for $h K 1$ in relation to the total section area and (2) the intensity of the staining (Figure 2C and D). As shown in Figure 2E, ANOVA detected a difference in hK1 expression with regard to the tumour location $(P=0.02)$, with largest positive areas in small intestine samples $(18.6 \pm 4.7 \%$ of total section), followed by the stomach $(9.1 \pm 2.4 \%)$ and rectum $(2.9 \pm 2.2 \%)$. In contrast, hK1 expression was independent of the risk index or cellular type (Figure $2 \mathrm{~F}$ and $\mathrm{G}$ ). Furthermore, no correlation was found with the tumour vascular density, adjusting for age, sex, tumour cellular type, location and risk index.

\section{GIST cell lines express hK1}

Two different GIST cell lines were analysed for hK1 expression using RT - PCR and ELISA. Both Imatinib-sensitive GIST882 cells and Imatinib-resistant GIST48 express hK1 mRNA and protein. In particular, GIST 882 cells release $30-400 \mathrm{pg} \mathrm{ml}^{-1}$ and GIST48 cells about $40 \mathrm{pg} \mathrm{ml}^{-1}$ of immunoreactive hK1 into the culture medium. Furthermore, the exposure of GIST882 cells to hypoxia and serum deprivation increased hK1 mRNA levels (Figure 3A) and immunoreactive hK1 content in the culture medium (Figure 3B). To verify that secreted hK1 is enzymatically active, GIST882 supernatants were assayed using a colorimetric assay for hK1 in the presence or absence of the serine protease inhibitor aprotinin
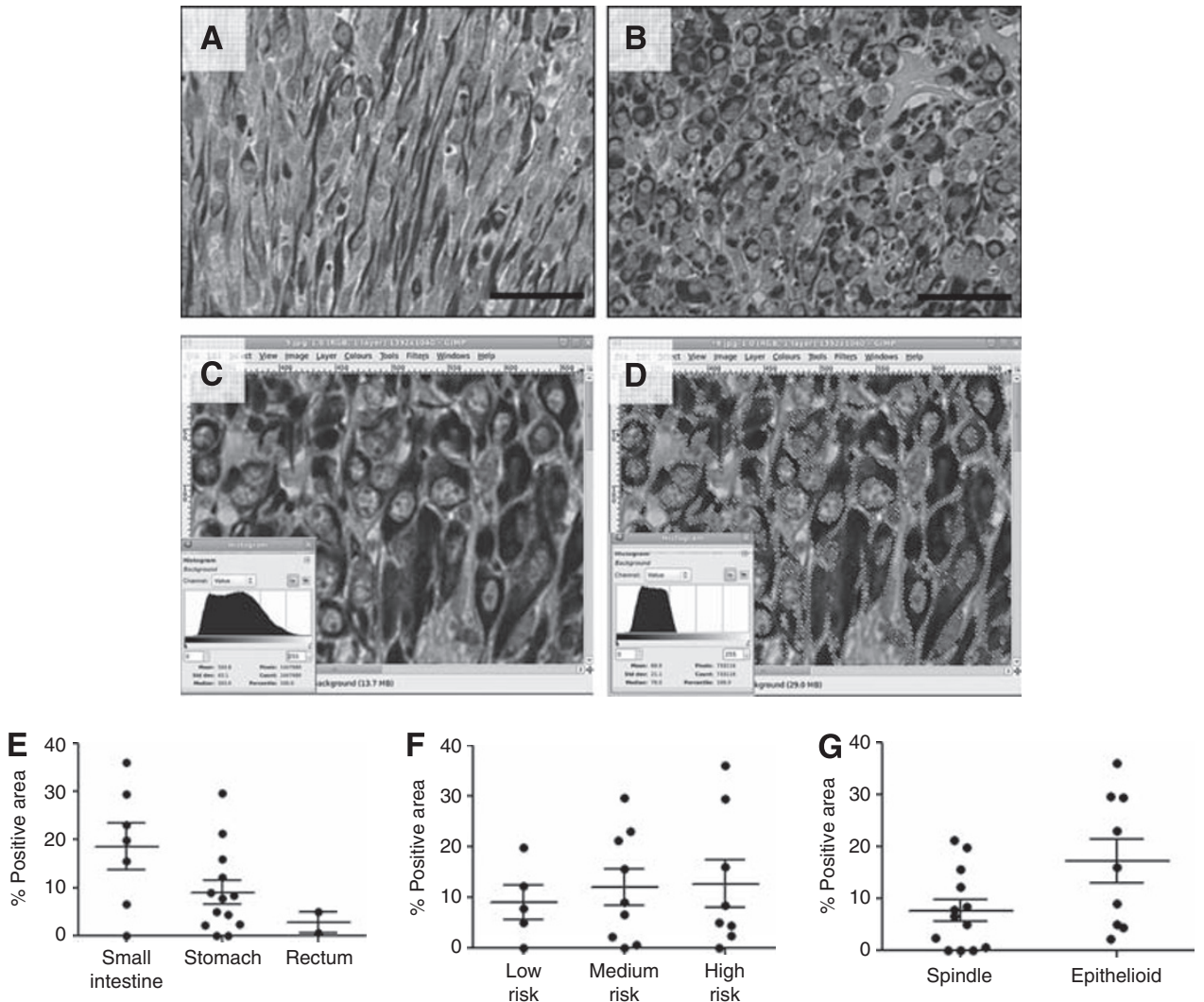

Figure 2 Immunohistochemical localisation of hKI in human GIST samples. Microphotographs show strong cytoplasmatic staining for hKI in the tumour tissue in both spindle-shaped $(\mathbf{A})$ and epithelioid cells $(\mathbf{B})$. Scale bar: $100 \mu \mathrm{m}$. For each section, the area positive for hKI was selected and the ratio between the pixels of the selected area and total number of pixels was calculated (in the example, 733 I I 6/I $447680 \times$ I00=56\%). Histograms show the analysis of corresponding images. Average colour intensity in the selection was considered as staining intensity (median $=70$ in the bottom right histogram $\mathbf{C}$ and $\mathbf{D}$ ). Scatterplot graphs showing the relationship between the calculated expression of hKI and GIST localisation (E), risk $(\mathbf{F})$ and cell type $(\mathbf{G})$. Each dot represents a GIST case. 

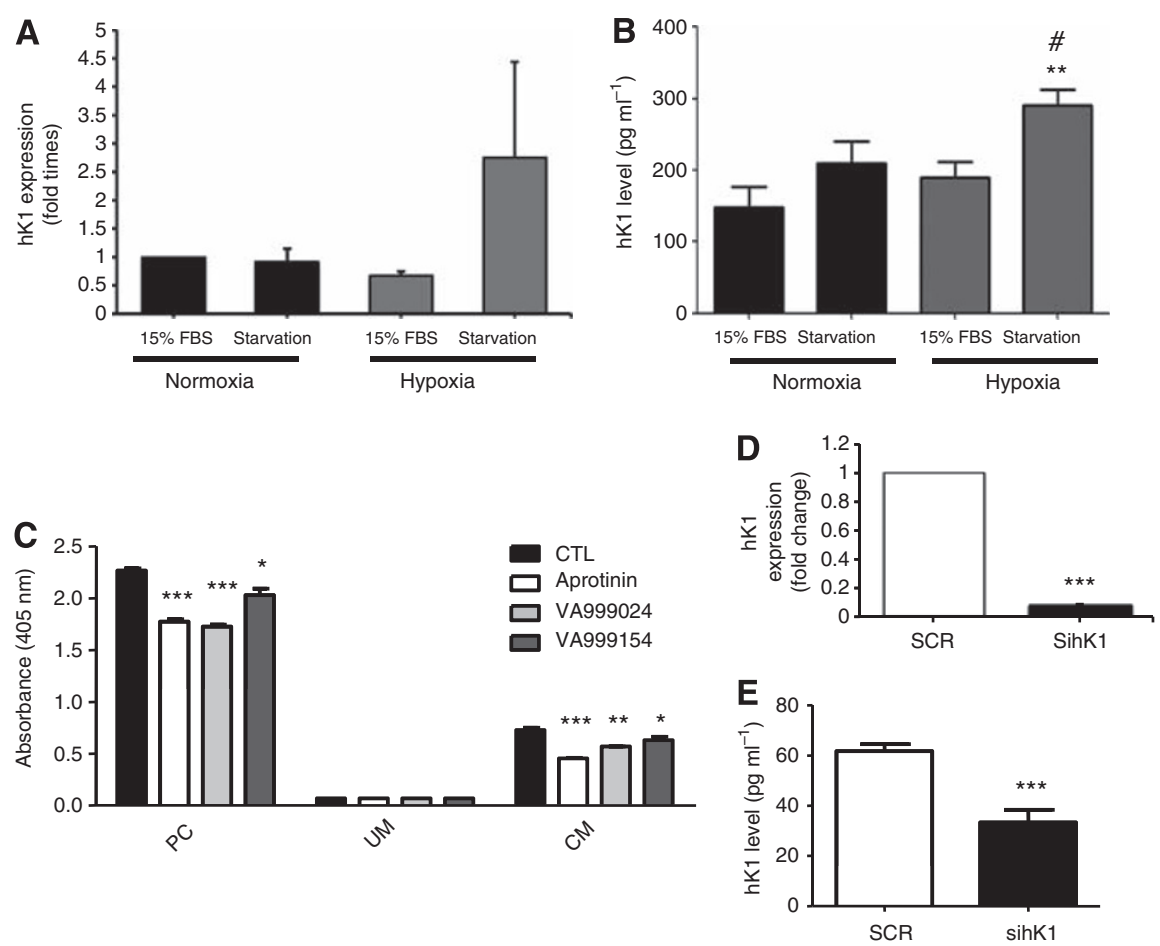

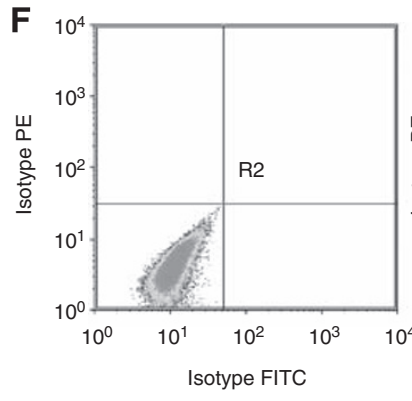

G

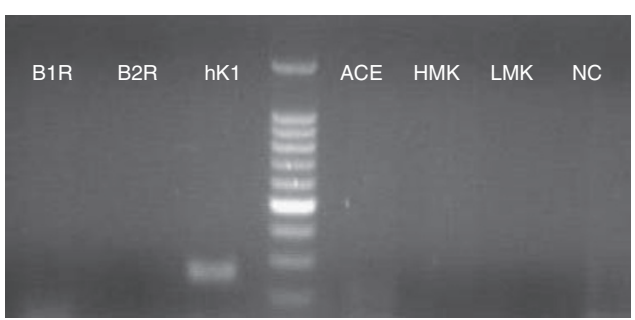

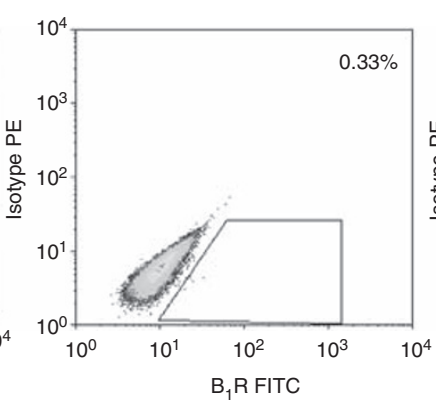

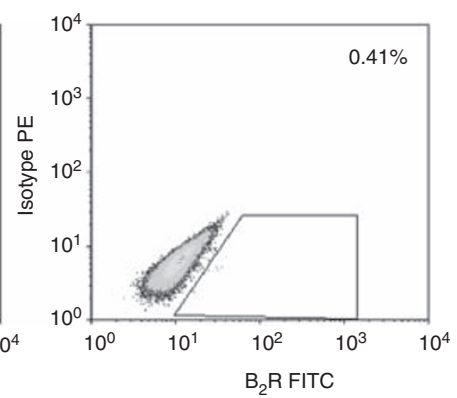

H

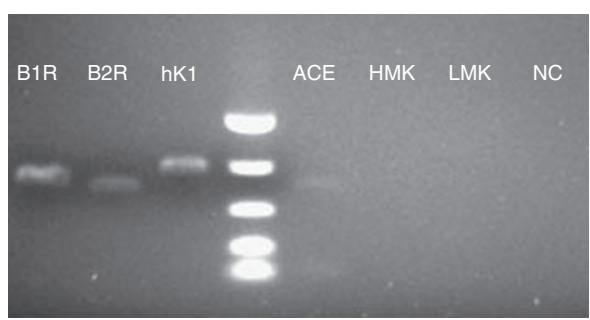

Figure 3 Gastrointestinal stromal tumour cell expression of kallikrein system components. Bar graphs show the effect of hypoxia and serum deprivation (starvation) on hKI mRNA (A) and protein secretion into culture medium (B). HKI mRNA was normalised for I8S and expressed as fold change vs I5\% FBS. Values are mean \pm s.e.m. of $n=6$ replicates. $* * P<0.01$ vs normoxia $+15 \%$ FBS andP $<0.05$ vs hypoxia $+15 \%$ FBS. (C) GIST882 cells release enzymatically active hKI in the conditioned medium (CM). Urine was used as a positive control (PC). Unconditioned medium (UM) was included as a negative control. Values are mean \pm s.e.m. of two measurements. $* P<0.05$, $* * P<0.0$ I $* * * P<0.00$ I vs non-inhibited reaction ( $C T L$ ). Effective hKI silencing (sihKI) was verified by RT-PCR (D) and ELISA on conditioned media (E). Human tissue kallikrein mRNA was normalised for I8S and expressed as fold change vs SCR. Values are representative of three independent experiments. $* * * *<0.001$ vs respective control. SCR $=$ scramble. Flow cytometry analyses show that GIST882 cells do not express $B_{1} R$ and $B_{2} R$, angiotensin-converting enzyme (ACE) or low (LMK) and high-molecular weight kininogen (HMK) (F and $\mathbf{G})$. GIST48 RT-PCR analysis shows expression of both kinin receptors, hKI and ACE (H). Negative control (NC) is the reaction run without cDNA.

and specific inhibitors VA999154 and VA999024. Results showed the presence of inhibitable enzymatic activity in GIST882 supernatants (Figure 3C). Lower levels of hK1 expression detected in GIST48 supernatants were not sufficient for the enzymatic activity quantification (not shown). Levels of hK1 mRNA and protein could be efficiently inhibited using siRNA (Figure 3D and E).

\section{Effect of hK1 on GIST882 cell function}

Human tissue kallikrein might be implicated in GIST growth and invasiveness through an autocrine mechanism mediated by kinin receptors, as well as through promotion of extracellular matrix degradation. 
A $\mathrm{i}$

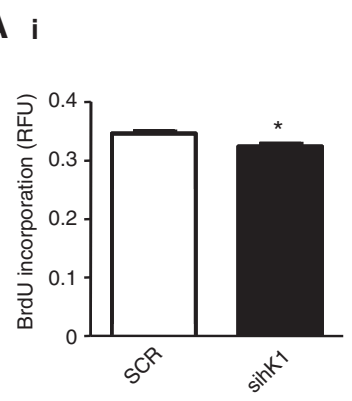

ii

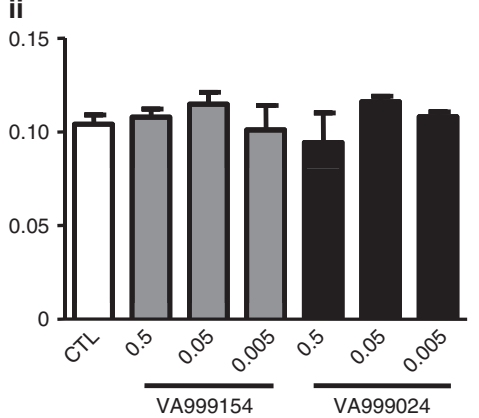

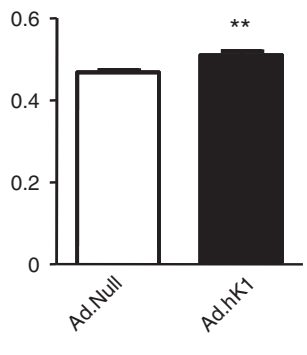

B
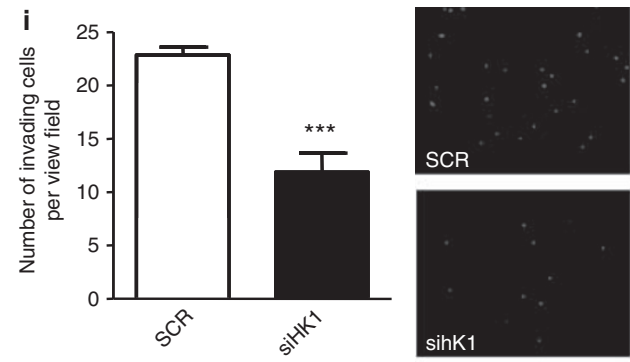

ii
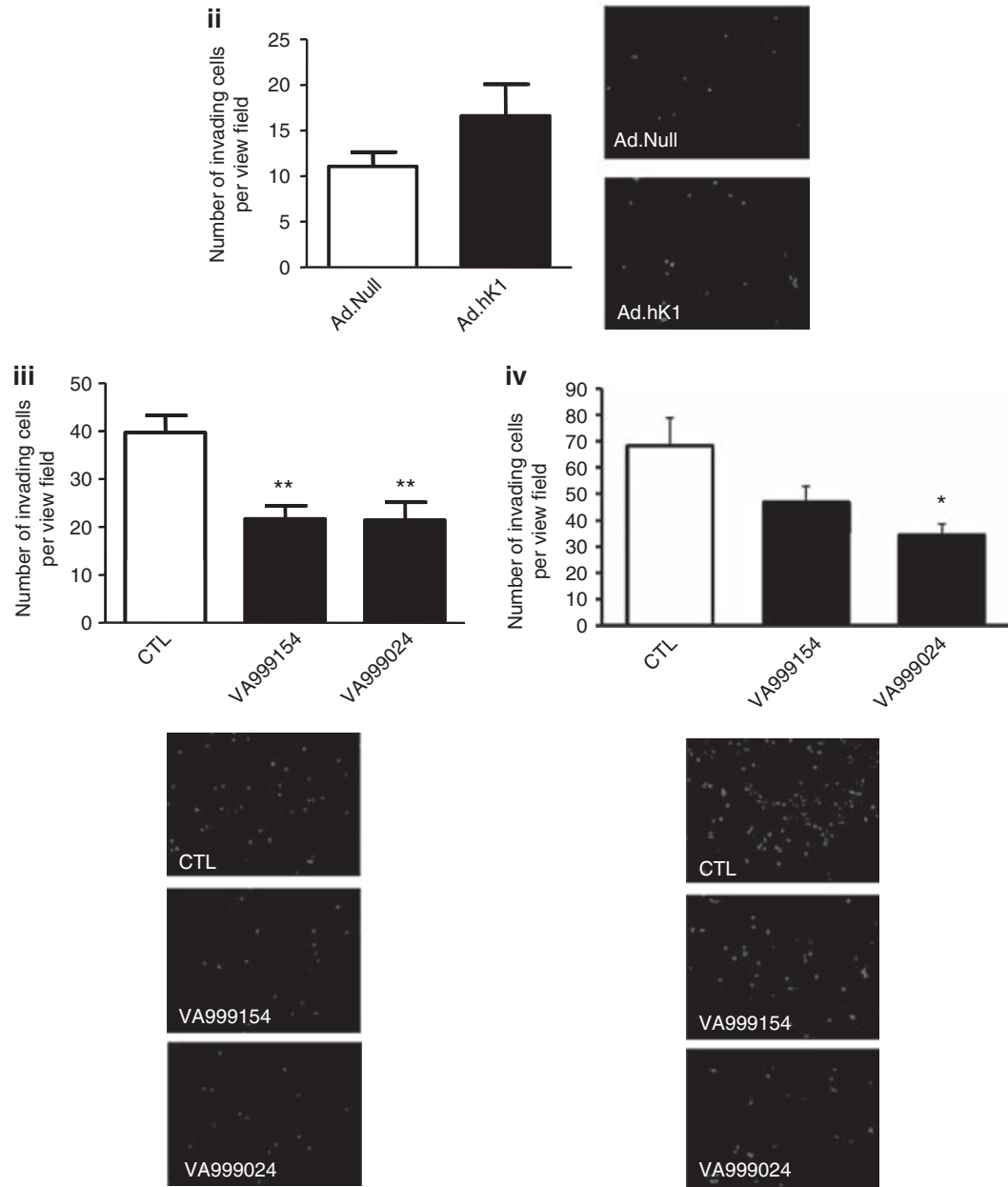

Figure 4 Human tissue kallikrein is implicated in the invasive capacity of GIST cells. Gene silencing by sihKI or forced expression of hKI by Ad.hKI produced mild reciprocal effects on GIST882 cell proliferation, whereas pharmacological inhibition was ineffective (Ai-Aiii). Invasion activity of GIST882 cells was strongly reduced by sihKI compared with scramble (SCR), conversely, infection with Ad.hKI enhanced GIST882 invasion as compared with Ad.Null (Bi and Bii). Pharmacological inhibition of hKI (VA999I54 and VA999024) significantly reduced GIST882 and GIST48 invasion capacity (Biii and Biv) Values are mean \pm s.e.m. of $n=3$ experiments, each performed in quadruplicate. $* P<0.05$, $* * * 0.0$ I and $* * * P<0.00$ I vs respective control (empty bar). 
Flow cytometry (Figure 3F) and RT - PCR (Figure 3G) demonstrated the absence of $B_{1} R$ and $B_{2} R$ protein and mRNA in GIST882 cells. Furthermore, GIST882 cells do not express kininogens or the kinin-degrading enzyme angiotensin-converting enzyme (ACE). Conversely, GIST48 showed expression of both receptors and ACE (Figure $3 \mathrm{H}$ ). These data indicate that, although hK1 is the only component of the kallikrein-kinin system expressed by GIST882 cells, therefore excluding kinin receptor-mediated autocrine mechanisms in these tumoural cells, GIST48 might respond directly to hK1.

The effect of changes in hK1 expression on GIST882 cell functional properties was then studied using siRNA-mediated silencing, inhibition by VA999154 and VA999024, or adenovirusmediated $h K 1$ gene transfer. Efficient transduction after gene transfer was confirmed by ELISA showing a $3 \times 10^{3}$-fold increase in hK1 levels compared with Ad.Null (data not shown).

A very small effect of hK1 silencing on GIST882 proliferation was detected (Figure 4Ai), but not confirmed, by pharmacological inhibition (Figure 4Aii). Similarly, a very small, but significant increase in GIST882 proliferation following hK1 transduction was observed (Figure 4Aiii). Pharmacological inhibition of hK1 did not exert significant effects on GIST48 proliferation (data not shown).

Silencing of hK1 significantly decreased, whereas hK1 overexpression tended to increase GIST 882 cell invasive capacity
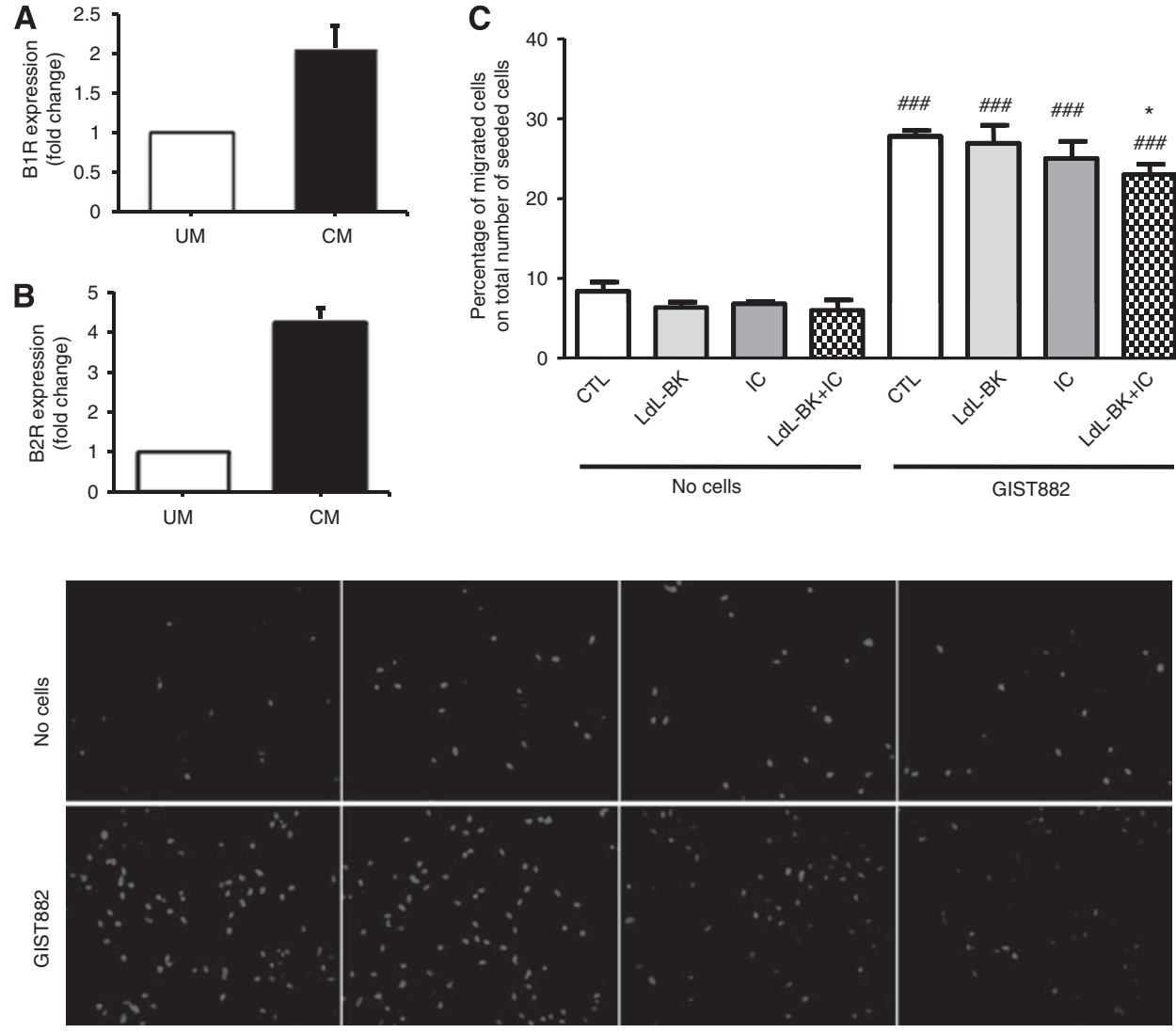

CTL

LdL-BK

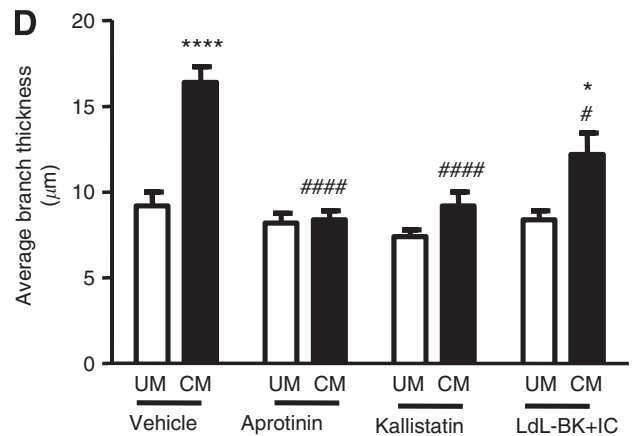

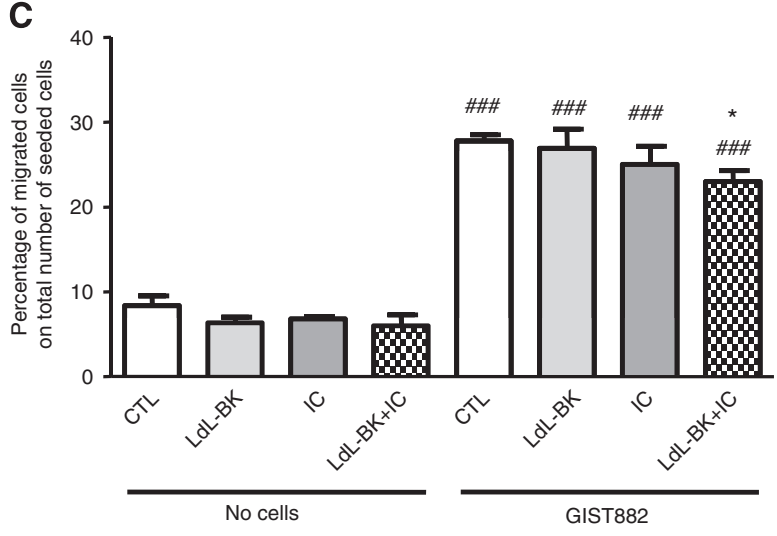

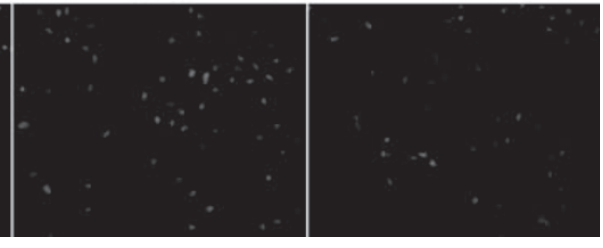

IC

LdL-BK+IC

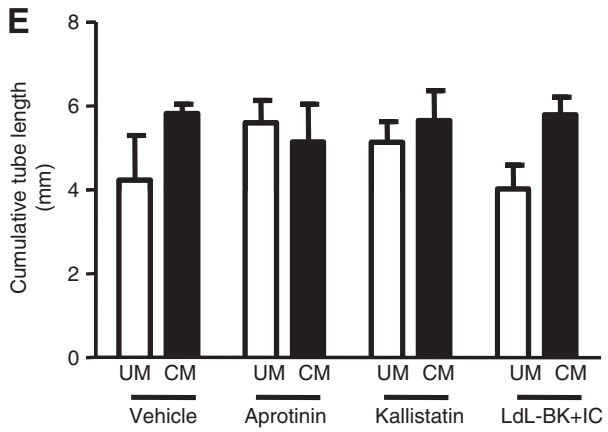

Figure 5 GIST882 cells stimulate endothelial cell migration and network formation capacity by a mechanism involving hKI and kinin receptors. Bar graphs showing the expression levels of $B_{1} R(\mathbf{A})$ and $B_{2} R(\mathbf{B})$ in HUVEC incubated with GIST882-conditioned medium (CM) or unconditioned medium (UM). Values were normalised for I8S and expressed as fold change vs UM. Bar graph shows the effect of GIST882 cells on HUVEC migration capacity. The presence of GIST in the bottom chamber enhanced HUVEC migration. Addition of $B_{1} R$ (Lys-des-Arg ${ }^{9}$ Leu $^{8}-B K$, LdL-BK) and B $R$ R (Icatibant, IC) antagonists did not have any effect on spontaneous migration as compared with the control (CTL), but reduced GIST-dependent migration (C). Bar graph shows the effect of GIST882-conditioned medium (CM) as compared with unconditioned medium (UM) on tube width (D) and tube length (E) in networks formed by HUVEC on Matrigel, in the presence or absence of aprotinin, kallistatin of kinin receptor antagonists. Values are mean \pm s.e.m. of six measures. $\# \# P<0.00$ I vs no cells or vehicle; $* P<0.05$ and ${ }^{*} * * * P<0.001$ vs vehicle or UM. 
(Figure $4 \mathrm{Bi}$ and Bii). Pharmacological inhibition of hK1 reduced both GIST882 and GIST48 invasiveness, suggesting that hK1 might be involved in GIST dissemination (Figure 4Biii and Biv).

\section{Endothelial cell migration towards GIST is mediated by kinin receptors}

Previous studies highlighted the role of endogenous and transgenic $\mathrm{hK} 1$ in the promotion of angiogenesis (Emanueli et al, 2000, 2001; Stone et al, 2009). Here, we assessed whether GIST-released hK1 may exert a proangiogenic action on HUVEC, thus supporting an attractive effect on host vessels.

Exposure of HUVECs to GIST882-conditioned medium increased $B_{2} R$ mRNA expression by four-fold, and $B_{1} R$ by two-fold (Figure $5 \mathrm{~A}$ and $\mathrm{B}$ ). In a transwell migration assay, GIST882 cells strongly attracted HUVECs. Addition of $\mathrm{B}_{1} \mathrm{R}$ or $\mathrm{B}_{2} \mathrm{R}$ antagonists had only a mild effect on HUVEC migration, whereas a $25 \%$ inhibition was obtained with the combination of both compounds (Figure $5 \mathrm{C}$ ). These data suggest that $\mathrm{B}_{1} \mathrm{R}$ and $\mathrm{B}_{2} \mathrm{R}$ are involved in GIST-induced HUVEC migration.

We then verified whether GIST882-conditioned medium has a stimulatory action on HUVEC network formation capacity in the Matrigel assay. We found that GIST882-conditioned medium stimulated the formation of wider branches, with this effect being inhibited by aprotinin, the hK1 inhibitor kallistatin and, to a minor extent, the combination of $B_{1} R$ and $B_{2} R$ antagonists (Figure 5D). In contrast, GIST882-conditioned medium did not influence the cumulative length of branches (Figure 5E). These results indicate that hK1 secreted by GIST contributes to endothelial migration and promotes vascular stabilisation.

\section{Local and systemic expression of $\mathrm{hK} 1$ in mice with xenogeneic GIST graft}

Finally, we tested whether xenogeneic GIST882 cell grafts in nude mice reproduce the phenotype observed in GIST-bearing patients, for example, local expression of hK1 and high hK1 circulating levels.

To this aim, three escalating GIST882 cell dosages $\left(3 \times 10^{6}\right.$, $5 \times 10^{6}$ and $8 \times 10^{6}$ ) or vehicle was implanted under the skin of $\mathrm{BALB} / \mathrm{c}$ nude mice. Tumour growth was directly proportional to the number of injected cells, with the lowest cell dosage $\left(3 \times 10^{6}\right)$ barely forming identifiable nodules (Figure 6A). Similarly, circulating levels of hK1 increased in tumour-bearing mice in proportion to tumour size, being detectable even in the smallest dosage of GIST 882 cells (Figure 6B). Tumours were explanted from mice given $5 \times 10^{6}$ or $8 \times 10^{6}$ GIST882 cells and subjected to verification of hK1 expression by ELISA and immunohistochemistry. Enzyme-linked immunosorbent assay revealed high levels of hK1 in the examined tumours (Figure 6C). Immunohistochemistry confirmed the cytoplasmatic staining for hK1 as observed in human GIST sections (Figure 6D). In particular, hK1 was expressed in the central core and the peripheral part of the tumour. Analysis of tumour vasculature identified host vessels invading the periphery of the xenograft (Figure 6E).

\section{DISCUSSION}

A significant number of adenocarcinomas express hKs. These tumours likely take advantage of hKs enzymatic properties to influence growth and metastasis (Borgono and Diamandis, 2004). Despite its early discovery, association of hK1 with cancer was late in comparison with other members of the hK family. A previous study showed the expression and localisation of hK1 in pancreatic adenocarcinoma of ductal origin and in a breast cancer cell line (Wolf et al, 2001). To the best of our knowledge, this is the first documentation that GISTs, the most common mesenchymal
A

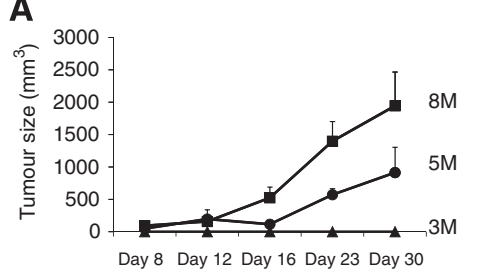

B
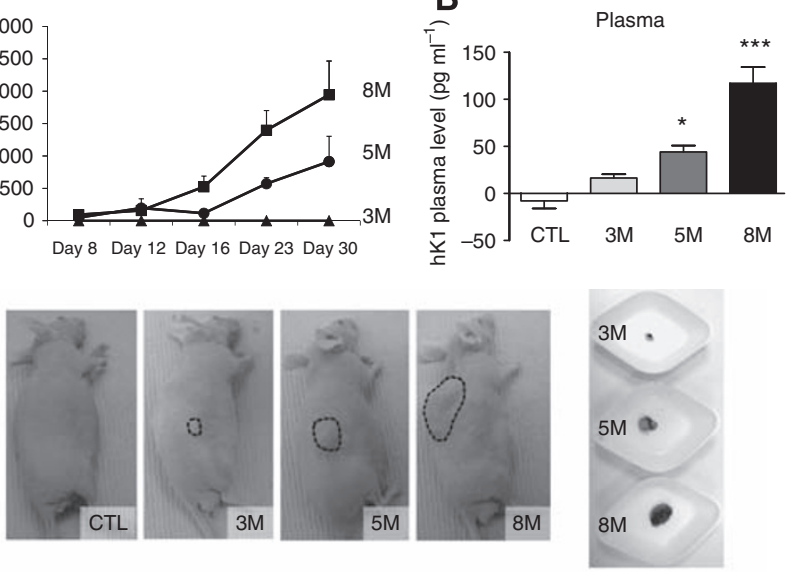

C
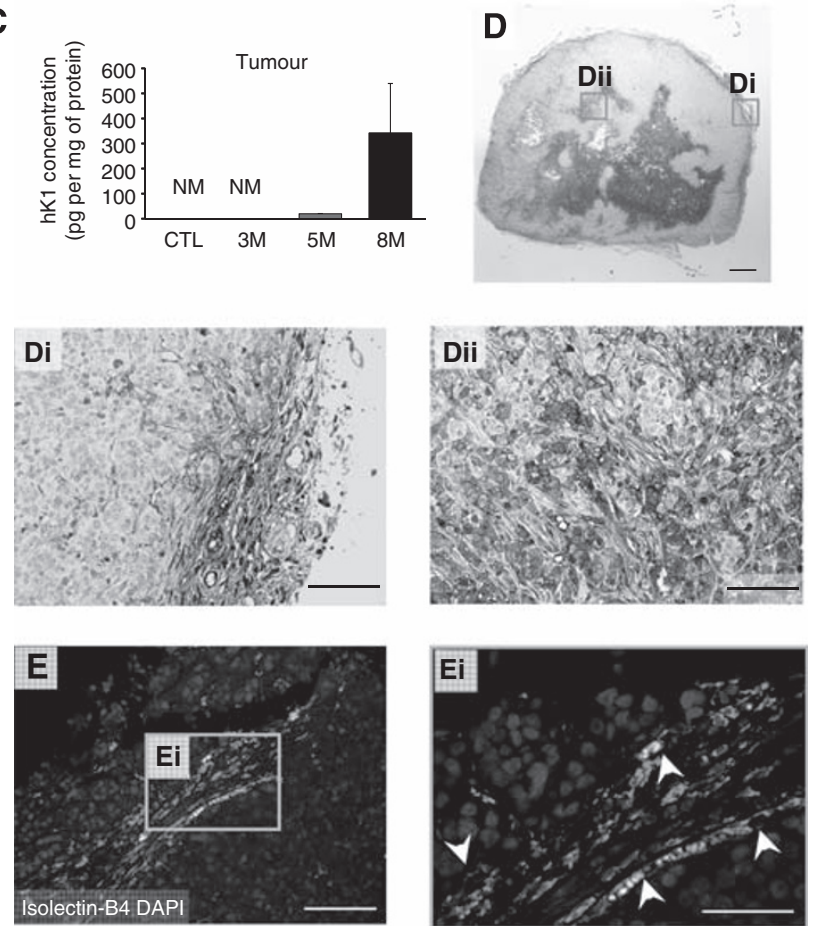

Figure 6 Expression of hKI by GIST xenografts. Line graph showing the growth of GIST xenografts implanted in nude mice $(\mathbf{A})$. Three cell dosages were injected $\left(3 \times 10^{6}(3 \mathrm{M}), 5 \times 10^{6}(5 \mathrm{M})\right.$ and $8 \times 10^{6}(8 \mathrm{M})$ cells or vehicle; four mice per dosage). Pictures show the appearance of tumour masses. Although no apparent growth or small nodules were observed with the lower dosage, higher dosages produced increasingly large masses. Bar graphs show the levels of immunoreactive hKI in peripheral blood (B) and tumour nodules $(\mathbf{C})$. NM= not measurable. Microphotographs show hKl-positive cells in tumour nodules (D). Microphotograph shows host-derived endothelial cells stained by Isolectin B4 (red, E) at the periphery of the tumour forming vascular structures (Ei, white arrowheads). Green staining is due to red blood cell autofluorescence. ${ }^{*} P<0.05$ and $* * * P<0.001$ vs vehicle (empty bar). Scale bar: $500 \mu \mathrm{m}$ (D), $100 \mu \mathrm{m}$ (Di-ii and $\mathbf{E})$ and $50 \mu \mathrm{m}(\mathbf{E i})$.

tumour of the gastrointestinal tract, express and release hK1 into the surrounding environment and circulation. The reason for this aberrant expression remains unknown. HK1 expression is responsive to oestrogens (Madeddu et al, 1991); however, we could not find a gender-related difference in hK1 levels in the GISTs of our series. Furthermore, hK1 levels were not associated with the cellular type and indexes of aggressiveness. An association was instead found with tumour location, with the highest values in 
GISTs of the small intestine. Our in vitro data show that expression levels in the GIST cell line are increased by hypoxia/starvation, pointing to the possibility that areas of the tumour far away from the vasculature could be stimulated to produce larger amounts of $\mathrm{hK} 1$. This is, at least in part, in line with the prevalent localization of hK1 in the central avascular core of GIST882 xenografts.

In vitro studies on two different cell lines derived from Imatinibresistant or Imatinib-sensitive tumours showed the common expression of hK1. Cell biology results suggest two distinct molecular mechanisms by which hK1 could be implicated in GIST growth: (1) invasion through degradation of the ECM and (2) induction/stabilisation of host-derived tumour vasculature. A direct action of $\mathrm{hK} 1$ as an ECM-degrading enzyme could be complemented by the ability of hK1 to cleave and activate other proteases, such as pro-MMPs (Menashi et al, 1994). The proinvasive action was verified by a gene titration approach, using siRNA to decrease native hK1 expression and $A d . h K 1$ to force hK1 production. Silencing resulted in inhibition of GIST invasive capacity and overexpression enhanced it. The effect of silencing was further confirmed using inhibitors of hK1 activity. It is therefore likely that changes in hK1 production, release and clearance by endogenous inhibitors may confer a different invasive profile to the tumour.

Coculture experiments showed that GIST882 cells exert an attractive action on HUVEC, which is at least in part due to activation of kinin receptors, as verified by the use of receptor antagonists. Furthermore, GIST882-conditioned medium stimulates HUVEC to form more robust branches than those observed using unconditioned medium. The fact that kallistatin, which rapidly binds hK1 and inhibits its activity in vitro (Zhou et al, 1992), blocks the strengthening action of GIST882 cells on HUVEC networks argues in favour of $\mathrm{hK} 1$ as a promoter of cancer angiogenesis. Kallistatin itself was previously identified as an inhibitor of angiogenesis in gastric carcinomas (Zhu et al, 2007). In the retrospective analysis of human GISTs, we could not find any correlation between hK1 expression and vascular density, suggesting that other mechanisms may overwhelm or confound the proangiogenic effect of hK1 in vivo. On the other side, although HUVECs are widely used as models for tumoural angiogenesis, different mechanisms might drive cancer endothelial cells.

In conclusion, results of our study show for the first time that hK1 is implicated in GIST invasion and angiogenesis. GIST882 xenografts express and release hK1 into the circulation, a result that calls for further validation of $\mathrm{hK} 1$ as a potential diagnostic biomarker. On a therapeutic standpoint, hK1 inhibitors showed promising results in models of breast cancer invasion (Wolf et al, 2001). Combination therapies incorporating hK1 inhibition/silencing as adjuvant to imatinib mesylate therapy may be useful for the treatment of GIST.

\section{ACKNOWLEDGEMENTS}

P Dominek was supported by a studentship of the Pathological Society of Great Britain and Ireland. We are thankful to Dr J Fletcher, Dr S Bauer and J Ketzer for providing cell lines GIST882 and GIST48.

\section{REFERENCES}

Bhoola K, Ramsaroop R, Plendl J, Cassim B, Dlamini Z, Naicker S (2001) Kallikrein and kinin receptor expression in inflammation and cancer. Biol Chem 382: $77-89$

Biyashev D, Tan F, Chen Z, Zhang K, Deddish PA, Erdos EG, Hecquet C (2006) Kallikrein activates bradykinin B2 receptors in absence of kininogen. Am J Physiol 290: H1244-H1250

Borgono CA, Diamandis EP (2004) The emerging roles of human tissue kallikreins in cancer. Nat Rev 4: 876-890

Chee J, Naran A, Misso NL, Thompson PJ, Bhoola KD (2008) Expression of tissue and plasma kallikreins and kinin B1 and B2 receptors in lung cancer. Biol Chem 389: 1225-1233

Clements JA, Willemsen NM, Myers SA, Dong Y (2004) The tissue kallikrein family of serine proteases: functional roles in human disease and potential as clinical biomarkers. Crit Rev Clin Lab Sci 41: 265-312

Demetri GD, von Mehren M, Blanke CD, Van den Abbeele AD, Eisenberg B, Roberts PJ, Heinrich MC, Tuveson DA, Singer S, Janicek M, Fletcher JA, Silverman SG, Silberman SL, Capdeville R, Kiese B, Peng B, Dimitrijevic S, Druker BJ, Corless C, Fletcher CD, Joensuu H (2002) Efficacy and safety of imatinib mesylate in advanced gastrointestinal stromal tumors. $N$ Engl J Med 347: 472-480

Desrivieres S, Lu H, Peyri N, Soria C, Legrand Y, Menashi S (1993) Activation of the $92 \mathrm{kDa}$ type IV collagenase by tissue kallikrein. J Cell Physiol 157: $587-593$

Dlamini Z, Bhoola KD (2005) Upregulation of tissue kallikrein, kinin B1 receptor, and kinin $\mathrm{B} 2$ receptor in mast and giant cells infiltrating oesophageal squamous cell carcinoma. J Clin Pathol 58: 915-922

Dlamini Z, Raidoo D, Bhoola K (1999) Visualisation of tissue kallikrein and kinin receptors in oesophageal carcinoma. Immunopharmacology 43: $303-310$

Emanueli C, Minasi A, Zacheo A, Chao J, Chao L, Salis MB, Straino S, Tozzi MG, Smith R, Gaspa L, Bianchini G, Stillo F, Capogrossi MC, Madeddu P (2001) Local delivery of human tissue kallikrein gene accelerates spontaneous angiogenesis in mouse model of hindlimb ischemia. Circulation 103: $125-132$

Emanueli C, Salis MB, Van Linthout S, Meloni M, Desortes E, Silvestre JS, Clergue M, Figueroa CD, Gadau S, Condorelli G, Madeddu P (2004) Akt/protein kinase $\mathrm{B}$ and endothelial nitric oxide synthase mediate

muscular neovascularization induced by tissue kallikrein gene transfer Circulation 110: $1638-1644$

Emanueli C, Zacheo A, Minasi A, Chao J, Chao L, Salis MB, Stacca T, Straino S, Capogrossi MC, Madeddu P (2000) Adenovirus-mediated human tissue kallikrein gene delivery induces angiogenesis in normoperfused skeletal muscle. Arterioscler Thromb Vasc Biol 20: 2379-2385

Fletcher CD, Berman JJ, Corless C, Gorstein F, Lasota J, Longley BJ, Miettinen M, O'Leary TJ, Remotti H, Rubin BP, Shmookler B, Sobin LH, Weiss SW (2002) Diagnosis of gastrointestinal stromal tumors: a consensus approach. Hum Pathol 33: 459-465

Heinrich MC, Griffith DJ, Druker BJ, Wait CL, Ott KA, Zigler AJ (2000) Inhibition of c-kit receptor tyrosine kinase activity by STI 571, a selective tyrosine kinase inhibitor. Blood 96: 925-932

Hirota S, Isozaki K, Nishida T, Kitamura Y (2000) Effects of loss-offunction and gain-of-function mutations of c-kit on the gastrointestinal tract. J Gastroenterol 35(Suppl 12): $75-79$

Leeb-Lundberg LM, Marceau F, Muller-Esterl W, Pettibone DJ, Zuraw BL (2005) International union of pharmacology. XLV. Classification of the kinin receptor family: from molecular mechanisms to pathophysiological consequences. Pharmacol Rev 57: $27-77$

Madeddu P, Emanueli C, El-Dahr S (2007) Mechanisms of disease: the tissue kallikrein-kinin system in hypertension and vascular remodeling. Nat Clin Pract 3: 208-221

Madeddu P, Gherli T, Bacciu PP, Maioli M, Glorioso N (1993) A kallikreinlike enzyme in human vascular tissue. Am J Hypertens 6: 344-348

Madeddu P, Glorioso N, Maioli M, Demontis MP, Varoni MV, Anania V, Xiong W, Chai K, Chao J (1991) Regulation of rat renal kallikrein expression by estrogen and progesterone. J Hypertens Suppl 9: S244-S245

Menashi S, Fridman R, Desrivieres S, Lu H, Legrand Y, Soria C (1994) Regulation of $92-\mathrm{kDa}$ gelatinase $\mathrm{B}$ activity in the extracellular matrix by tissue kallikrein. Ann N Y Acad Sci 732: 466-468

Miettinen M, Lasota J (2006) Gastrointestinal stromal tumors: review on morphology, molecular pathology, prognosis, and differential diagnosis. Arch Pathol Lab Med 130: 1466-1478

Porcu P, Emanueli C, Desortes E, Marongiu GM, Piredda F, Chao L, Chao J, Madeddu P (2004) Circulating tissue kallikrein levels correlate 
with severity of carotid atherosclerosis. Arterioscler Thromb Vasc Biol 24: $1104-1110$

Porcu P, Emanueli C, Kapatsoris M, Chao J, Chao L, Madeddu P (2002) Reversal of angiogenic growth factor upregulation by revascularization of lower limb ischemia. Circulation 105: 67-72

Sawant S, Snyman C, Bhoola K (2001) Comparison of tissue kallikrein and kinin receptor expression in gastric ulcers and neoplasms. Int Immunopharmacol 1: $2063-2080$

Stone OA, Richer C, Emanueli C, van Weel V, Quax PH, Katare R, Kraenkel N, Campagnolo P, Barcelos LS, Siragusa M, Sala-Newby GB, Baldessari D, Mione M, Vincent MP, Benest AV, Al Haj Zen A, Gonzalez J, Bates DO, Alhenc-Gelas F, Madeddu P (2009) Critical role of tissue kallikrein in vessel formation and maturation: implications for therapeutic revascularization. Arterioscler Thromb Vasc Biol 29: $657-664$

Tschesche H, Michaelis J, Kohnert U, Fedrowitz J, Oberhoff R (1989) Tissue kallikrein effectively activates latent matrix degrading metalloenzymes. Adv Exp Med Biol 247A: 545-548

Tuveson DA, Willis NA, Jacks T, Griffin JD, Singer S, Fletcher CD, Fletcher JA, Demetri GD (2001) STI571 inactivation of the gastrointestinal stromal tumor c-KIT oncoprotein: biological and clinical implications. Oncogene 20: $5054-5058$
Wang C, Chao L, Chao J (1995) Direct gene delivery of human tissue kallikrein reduces blood pressure in spontaneously hypertensive rats. J Clin Investig 95: $1710-1716$

Welch HG, Albertsen PC (2009) Prostate cancer diagnosis and treatment after the introduction of prostate-specific antigen screening: 1986-2005. J Natl Cancer Inst 101: 1325 - 1329

Wolf WC, Evans DM, Chao L, Chao J (2001) A synthetic tissue kallikrein inhibitor suppresses cancer cell invasiveness. Am J Pathol 159: 1797 - 1805

Wolf WC, Harley RA, Sluce D, Chao L, Chao J (1999) Localization and expression of tissue kallikrein and kallistatin in human blood vessels. J Histochem Cytochem 47: $221-228$

Yao YY, Yin H, Shen B, Smith Jr RS, Liu Y, Gao L, Chao L, Chao J (2008) Tissue kallikrein promotes neovascularization and improves cardiac function by the Akt-glycogen synthase kinase-3beta pathway. Cardiovasc Res 80: $354-364$

Zhou GX, Chao L, Chao J (1992) Kallistatin: a novel human tissue kallikrein inhibitor. Purification, characterization, and reactive center sequence. J Biol Chem 267: 25873-25880

Zhu B, Lu L, Cai W, Yang X, Li C, Yang Z, Zhan W, Ma JX, Gao G (2007) Kallikrein-binding protein inhibits growth of gastric carcinoma by reducing vascular endothelial growth factor production and angiogenesis. Mol Cancer Therap 6: 3297-3306 